\title{
Fasting and engineered diets as powerful tool in the medical practice: an old approach in the new era
}

\author{
Concetta Panebianco ${ }^{1}$, Adele Potenza ${ }^{2}$, Valerio Pazienza ${ }^{1}$ \\ ${ }^{1}$ Gastroenterology Unit, ${ }^{2}$ Clinical Nutrition Unit, IRCCS “Casa Sollievo della Sofferenza” Hospital, San Giovanni Rotondo (FG), Italy \\ Correspondence to: Dr. Valerio Pazienza, PhD. Gastroenterology Unit, IRCCS “Casa Sollievo della Sofferenza” Hospital, Viale dei Cappuccini, 1-71013 \\ San Giovanni Rotondo (FG), Italy. Email: pazienza_valerio@yahoo.it. \\ Provenance: This is a Guest Editorial commissioned by Section Editor Zhijun Han, MD (Department of Laboratory Medicine, Wuxi Second \\ Hospital, Nanjing Medical University, Wuxi, China). \\ Comment on: Wei M, Brandhorst S, Shelehchi M, et al. Fasting-mimicking diet and markers/risk factors for aging, diabetes, cancer, and cardiovascular \\ disease. Sci Transl Med 2017;9:eaai8700.
}

Submitted Aug 08, 2017. Accepted for publication Aug 21, 2017.

doi: $10.21037 /$ atm.2017.08.34

View this article at: http://dx.doi.org/10.21037/atm.2017.08.34

Fasting and dietary restriction have been practiced for millennia for different purposes spanning from religious, philosophical to medical. Only in recent times it has been scientifically demonstrated that dietary restriction is associated with major health benefits such as: amelioration of cardiovascular diseases, immune disorders, slowing of the aging processes, diabetes, insulin resistance and reduced risk of cancer (1). Moreover, cutting-edge studies, taking advantage of in vivo and in vitro models, unveiled a potential link between short-term starvation and improved efficacy of chemotherapy for some types of cancer $(2,3)$ accompanied by a decrease of side effects of chemotherapy in patients (4).

Previous animal studies by Dr. Longo's group, who is a pioneer in this field, have shown that fasting or diets that mimic fasting condition (FMD) extend lifespan, ameliorate cognitive functions, reduce inflammation and relieve the symptoms of diseases such as multiple sclerosis and cancer $(5,6)$. In a very recent study published by Dr. Longo and colleagues (7), a randomized controlled trial was conducted on humans, in order to evaluate the effects of a fastingmimicking diet on markers and risk factors for aging, cancer, metabolic syndrome and cardiovascular diseases. This study enrolled 100 healthy participants without a diagnosed medical condition in the previous 6 months, which were randomly assigned to either a control group maintaining a normal diet for 3 months or a FMD group following three consecutive cycles of a fasting-mimicking diet for 5 days/month. First interesting thing to consider is that the fasting-mimicking diet has been proved to be safe and produced no serious adverse effect. This represents a step ahead of fasting which can cause deleterious nutritional deficiencies, when prolonged, in spite of its potential beneficial health effects. At the end of the 3-month period, the participants belonging to FMD group presented reduced body weight, trunk and total body fat, lowered blood pressure and lower levels of the hormone insulin-like growth factor 1 (IGF-1), whose implication in aging and cancer is well documented (8-10). The participants of the control group were then shifted to the FMD regimen for another 3 months: a post hoc analysis was performed on both FMD arms, replicating the aforementioned results and moreover showing decreased body mass index, glucose, triglycerides, cholesterol, and C-reactive protein upon fasting-mimicking diet. The study by Longo and colleagues is undoubtedly well conceived and the results are exciting and encouraging. This work convincingly contributes to promote a healthier lifestyle in a society in which metabolic and aging-related diseases represent a major health burden. In industrialized countries, indeed, the diffusion of the Western diet (high in animal protein and fat, low in fiber) and of the "fast food culture" is responsible for high calorie intake (11) and, together with sedentary behavior, increases the risk of obesity, metabolic syndrome, cardiovascular diseases and cancer.

"A new food culture, based on limiting dietary surplus and on preferring healthier foods, is then essential."

However, it should be taken into account that in the 
aforementioned study, 71 out of the 100 participants recruited completed the trial till the end and it is worth to note that $25 \%$ of the participant who tested the fasting-mimicking diet and $10 \%$ belonging to the control group dropped out of the trial. As the authors themselves recognize, this difference in percentage between the two groups is likely due to the fact that, despite the efforts to reduce the burden of low-calorie/ protein diets, adherence to this low-calorie regimen requires commitment, power will and a strong mental discipline. This trial involved healthy people, some of which showing at the most risk factors for metabolic syndrome, but the authors envisage an application of this regimen also on patients with a diagnosed aging-related disease. Particular attention has to be paid when applying dietary interventions to diseased patients, especially in the frame of oncology. Patients may refuse to follow the dietary regimen due to their psychological status and fasting or fasting-mimicking diets may cause weight loss and muscle mass waste, thus worsening the cachexia syndrome, a condition which affects about half of all cancer patients. For these reasons, although dietary restriction is a powerful tool when used in experimental approaches, shifting this therapeutic method from animals to humans has to overcome the above mentioned difficulties. Therefore, alternative interventions would be desirable in order to take advantage of the benefits of calorie restriction while limiting negative consequences. Mathews and Liebenberg proposed the use of nutrient hemodialysis as a potential solution to achieve fasting "mechanically" (12) without requiring special waivers from patients. Another approach could consist in manipulating macronutrients in the diet without restricting energy intake (13). With the aim of decreasing the amount of glucose, which notoriously fuels cancer, ketogenic diets have been developed, characterized by high fat, moderate to low protein and very low carbohydrate content $(13,14)$. This kind of feeding, which forces the body to use fat rather than glucose as energy source, has proven to be effective as adjuvant in anticancer therapy in pre-clinical studies (14) and, although its efficacy is still being evaluated in human cancers, it seems feasible and safe in cancer patients (15). A further strategy to decrease glucose levels has been the development of engineered diets replacing corn starch with resistant starch, which is not digestible by the intestinal enzymes thus not releasing glucose. This approach has turned out to slow pancreatic cancer growth in a mouse model, without causing weight loss (16). Consistently, a number of previous studies on rat models of colon cancer revealed a protective role for resistant starch against carcinogenesis (17-20), even upon high-protein diets $(19,20)$.
Moreover, in very recent years, a growing body of evidence has been produced highlighting the prominent role of the diet in shaping gut microbiota composition, which in turn plays a major role in the pathogenesis of several diseases $(16,21)$. These studies suggest that not only the calories intake is important but also the type of dietary compounds. More studies on diet compounds and/or supplements are needed in order to better understand the relationship between diet and disease. It has been demonstrated that certain types of foods selectively enhance some microbial groups which in turn can shape the profile of the whole gut microbiota thus affecting the onset and the progression of several diseases, including cancer. Fiber-rich food regimens significantly decrease pro-inflammatory microorganisms such as Bacteroides acidifaciens, Escherichia coli, Ruminococcus gnavus and Clostridium cocleatum (16), supporting the indication that engineered diets able to perturb gut microbial community can be synergistically used together with the current chemotherapeutic approaches. Administration of probiotics and prebiotics represents the most common measure to shape gut microbiota in order to slow cancer progression, improve response to therapy or limit toxic side effects (22). As for cachexia, for instance, it has been recently demonstrated that administration of Lactobacillus reuteri in drinking water to mice with colon cancer predisposed to cachexia, has beneficial effects on gastrocnemius muscle masses and body weight that are increased as compared to untreated mice, together with reduced neutrophil counts, a marker of systemic inflammation (23).

In conclusion, in agreement with what has been stated elsewhere by Dr. Longo (24), dietary restriction effects on aging and health are not necessarily the result of a reduced caloric intake but also a consequence of changes in diet composition. Therefore, though recognizing the remarkable scientific value of the benefits provided by the fasting-mimicking diet, it is advisable to pursue less demanding strategies that would be more easily tolerated by healthy people and particularly by patients. Longo's studies undoubtedly play a pivotal role in the prevention and/or cure of aging-related diseases and also paved the way for further nutritional manipulations.

\section{Acknowledgements}

Funding: The study was supported by a grant from the Italian Ministry of Health through Division of Gastroenterology (RC1703GA31) of IRCCS Scientific Institute "Casa Sollievo della Sofferenza" Hospital, San 
Giovanni Rotondo (FG), Italy and by the " $5 \times 1000$ " voluntary contributions.

\section{Footnote}

Conflicts of Interest: The authors have no conflicts of interest to declare.

\section{References}

1. Lee C, Longo VD. Fasting vs dietary restriction in cellular protection and cancer treatment: from model organisms to patients. Oncogene 2011;30:3305-16.

2. Lee C, Raffaghello L, Brandhorst S, et al. Fasting cycles retard growth of tumors and sensitize a range of cancer cell types to chemotherapy. Sci Transl Med 2012;4:124ra27.

3. D'Aronzo M, Vinciguerra M, Mazza T, et al. Fasting cycles potentiate the efficacy of gemcitabine treatment in in vitro and in vivo pancreatic cancer models. Oncotarget 2015;6:18545-57.

4. Safdie FM, Dorff T, Quinn D, et al. Fasting and cancer treatment in humans: A case series report. Aging (Albany NY) 2009;1:988-1007.

5. Choi IY, Piccio L, Childress P, et al. A Diet Mimicking Fasting Promotes Regeneration and Reduces Autoimmunity and Multiple Sclerosis Symptoms. Cell Rep 2016;15:2136-46.

6. Brandhorst S, Choi IY, Wei M, et al. A Periodic Diet that Mimics Fasting Promotes Multi-System Regeneration, Enhanced Cognitive Performance, and Healthspan. Cell Metab 2015;22:86-99.

7. Wei M, Brandhorst S, Shelehchi M, et al. Fasting-mimicking diet and markers/risk factors for aging, diabetes, cancer, and cardiovascular disease. Sci Transl Med 2017;9:eaai8700.

8. Cheng CW, Adams GB, Perin L, et al. Prolonged fasting reduces IGF-1/PKA to promote hematopoietic-stem-cellbased regeneration and reverse immunosuppression. Cell Stem Cell 2014;14:810-23.

9. Sonntag WE, Lynch CD, Cefalu WT, et al. Pleiotropic effects of growth hormone and insulin-like growth factor (IGF)-1 on biological aging: inferences from moderate caloric-restricted animals. J Gerontol A Biol Sci Med Sci 1999;54:B521-38.

10. Dunn SE, Kari FW, French J, et al. Dietary restriction reduces insulin-like growth factor I levels, which modulates apoptosis, cell proliferation, and tumor progression in p53deficient mice. Cancer Res 1997;57:4667-72.

11. Tucker KL, Buranapin S. Nutrition and aging in developing countries. J Nutr 2001;131:2417S-23S.
12. Mathews EH, Liebenberg L. Short-term starvation for cancer control in humans. Exp Gerontol 2013;48:1293.

13. Klement RJ. Mimicking caloric restriction: what about macronutrient manipulation? A response to Meynet and Ricci. Trends Mol Med 2014;20:471-2.

14. Allen BG, Bhatia SK, Anderson CM, et al. Ketogenic diets as an adjuvant cancer therapy: History and potential mechanism. Redox Biol 2014;2:963-70.

15. Klement RJ. Beneficial effects of ketogenic diets for cancer patients: a realist review with focus on evidence and confirmation. Med Oncol 2017;34:132.

16. Panebianco C, Adamberg K, Adamberg S, et al. Engineered Resistant-Starch (ERS) Diet Shapes Colon Microbiota Profile in Parallel with the Retardation of Tumor Growth in In Vitro and In Vivo Pancreatic Cancer Models. Nutrients 2017;9:E331.

17. Le Leu RK, Hu Y, Young GP. Effects of resistant starch and nonstarch polysaccharides on colonic luminal environment and genotoxin-induced apoptosis in the rat. Carcinogenesis 2002;23:713-9.

18. Le Leu RK, Brown IL, Hu Y, et al. Suppression of azoxymethane-induced colon cancer development in rats by dietary resistant starch. Cancer Biol Ther 2007;6:1621-6.

19. Le Leu RK, Brown IL, Hu Y, et al. Effect of dietary resistant starch and protein on colonic fermentation and intestinal tumourigenesis in rats. Carcinogenesis 2007;28:240-5.

20. Toden S, Bird AR, Topping DL, et al. Differential effects of dietary whey, casein and soya on colonic DNA damage and large bowel SCFA in rats fed diets low and high in resistant starch. Br J Nutr 2007;97:535-43.

21. Singh RK, Chang HW, Yan D, et al. Influence of diet on the gut microbiome and implications for human health. J Transl Med 2017;15:73.

22. Zitvogel L, Galluzzi L, Viaud S, et al. Cancer and the gut microbiota: an unexpected link. Sci Transl Med 2015;7:271ps1.

23. Varian BJ, Goureshetti S, Poutahidis T, et al. Beneficial bacteria inhibit cachexia. Oncotarget 2016;7:11803-16.

24. Lee C, Longo V. Dietary restriction with and without caloric restriction for healthy aging. F1000Res 2016;5:F1000 Faculty Rev-117.

Cite this article as: Panebianco C, Potenza A, Pazienza V. Fasting and engineered diets as powerful tool in the medical practice: an old approach in the new era. Ann Transl Med 2017;5(21):429. doi: 10.21037/atm.2017.08.34 